www.jmscr.igmpublication.org

Index Copernicus Value: 79.54

ISSN (e)-2347-176x ISSN (p) 2455-0450

crossref DOI: https://dx.doi.org/10.18535/jmscr/v7i4.128

\title{
Infertility among Couples: It takes two for Procreation: A Review on Literature
}

\author{
Authors \\ Santhoshi Bollapragda ${ }^{1}$, Kadari Sunil ${ }^{2}$, B V Rama ${ }^{3}$ \\ ${ }^{1}$ MBBS (Taishan Medical University, China), ${ }^{2} \mathrm{MBBS}$ (IUHS), ${ }^{3}$ Andhra University, India
}

\begin{abstract}
Being a mother, both biologically and from inner emotions, is a very special journey and the same passes to a father too but there are many reasons behind not getting success rates in getting fertile still yet to be understood. Here in this paper, we have just gone through the literature survey of infertility and some of the reasons found in both female and male are presented in brief. In some of the cases, even doctors are unable to determine the cause of infertility when the required test results in both the male and female are normal but still, infertility prevails is unable to be explained by the doctors which makes the couple to struggle for a family. The person's mind, health issues, eating habits, the rise of moods and the body response to medicines as well as lifestyles are also to be considered to understand the reason for. People see infertility as a sickness, and so the very idea makes the person get frustrated and many people get disgusted and stop talking of what to do next to get success in their problem (infertility).
\end{abstract}

Keywords: Infertility, Biological, Fertile.

\section{Introduction}

The whole thing begins with the intercourse between a male and a female - reproductive process in which the penis is inserted into the vagina and through which a new human life may begin. Fertilization occurs when the union of the egg and sperm that takes place in the fallopian tubes, a baby comes to the world. According to Research conducted by Inito, a Bangalore-based medical technology company has unravelled some startling facts about infertility in India. According to the data, 10-15 percent, married couples in India face infertility. A whopping 27.5 million couples who want to conceive suffer from infertility. Nowadays, it is observed not only due to a clinical problem in the couple but focus on careers and the increase of late marriages at late ages also contribute. It is also worth adding that many studies emphasize how women seem more likely to be blamed for infertility, and to carry the greater burden of it (Macro, Organization, DHS, \& reports, 2004); ${ }^{[6],[44]}$.

\subsection{Factors effecting women}

Pregnancy, being a fortunate moment for personal and social transformation, inevitably changes the life of a woman, to the point of questioning her own identity and self-concept within the process of adaptation to a new condition and role. The way the pregnant woman experiences all changes and significant events requires in terms of development, an added effort, both physical and psychological, which is not always easy to manage, in order to prevent imbalances and emotional disturbances ${ }^{[14]}$. 
Pregnancy and Maternity are terms which are often mixed up and even used interchangeably, although they are two distinct realities, and consequently also with different and not always coincident psychological experience ${ }^{[35]}$. The first factor was designated family reasons, explains $23.91 \%$ of total variance, the second calculated factor was termed personal reasons, explains $16.89 \%$ of total variance and, finally, the third factor, entitled socioeconomic reasons which explain $14.16 \%$ of the total variance. (Ferreira et al., 2015). There is a relationship $(\mathrm{p}<0.05)$ between age, schooling, number of previous pregnancies, type of treatment used in case of missed abortion, duration of menstruation, infertility, and quality of life. Updating knowledge has become increasingly important for health professionals, aiming for the improvement of the quality of life of women confronted with a diagnosis of infertility. These results are consistent with those obtained by other studies that validate the change in the quality of life, according to several sociodemographic characteristics, obstetric history, and infertility diagnosis ${ }^{[14]}$. Mothering is, on the whole, is significant because it is the main vehicle through which people first form their identities and learn their place in society Multifaceted and complex, mothering is symbolically laden, representing what often is characterized as the ultimate in relational devotion Motherhood is entwined with notions of femininity [9], [17] and women's gender identity is reinforced by mothering ${ }^{[26]}$. A study on motherhood revealed that the past decade's advances that the study is significant, as is the fact that mothering is being studied from diverse perspectives and disciplines ${ }^{[35]}$. Mothering is associated with women because universally, it is women who do the work of mothering. Modern mothering entails extensive, ongoing emotional work ${ }^{[9],[43]}$. Feelings shift. There is no single emotion, love that children inspire in mothers, and feelings must be managed and directed. A mother's emotions can vary within the course of a day and certainly over time, depending upon the behavior of her children, space, time, and services available to her, and myriad other desires and frustrations ${ }^{\text {[33], [19]. }}$.

The factors for Motherhood is entwined with notions of femininity ${ }^{[9],[17]}$. and women's gender identity is reinforced by mothering ${ }^{[25]}$. A study on motherhood revealed that the past decade's advances that the study is significant, as is the fact that mothering are being studied from diverse perspectives and disciplines ${ }^{[1]}$, Modern mothering entails extensive, ongoing emotional ${ }^{[15],[9],}{ }^{[16]}$. Feelings shift. There is no single emotion, love that children inspire in mothers, and feelings must be managed and directed. A mother's emotions can vary within the course of a day and certainly over time, depending upon the behaviour of her children, space, time, and services available to her, and myriad other desires and frustrations see page $34^{[33] \text {, }}$ [19].

The factors for females considerate of the specific differences between fertile cycles and ovulatory cycles will in time assist the diagnosis and management of infertile patients, and we can find the gynaecological abnormality if exists. Another explanation, which is also difficult to prove, is that the presence within the reproductive tract of the preimplantation embryo has already influenced corpus luteum progesterone production in the conception cycles. The only other evidence that a preimplantation human embryo may influence the maternal organism is the demonstration of immune suppression by the rosette inhibition test ${ }^{[27]}$ Most studies have tried to capture pregnancy intention by asking women to classify their pregnancies into the distinct categories of 'wanted' and 'unwanted' with a further distinction within the unwanted category between those that were mistimed or untimed (wanted later) or unwanted (not wanted at all). For the purposes of analysis, many researchers group the unwanted together, although this risks the possibility of not being clear about the true effect of pregnancies that are never intended. The term 'unintended' to encompass pregnancies is both untimed and unwanted. Evidence from the US suggests that as many as 6 in 10 of these pregnancies end in abortion ${ }^{[2]}$. In women adhering 
correctly to contraceptive use, a frequency of sexual intercourse is a key factor in determining the chance of method failure. In clinical trials of women using the diaphragm those women who had more frequent intercourse were more likely to become pregnant despite perfect use than those having sex less often. Contraceptive failure rates decline with age and length of marriage in line with reduced coital frequency $^{[2]}$.

One most common factor is Fibroids, the symptoms include heavy and irregular menstrual bleeding with accompanying anaemia, pelvic pain, dysmenorrhoea, dyspareunia, increased urinary frequency, infertility, early pregnancy loss, they increase the production of angiogenic factors and a basic fibroblast growth factor and reduce the production of coagulation factors resulting in heavy menses. ${ }^{[7]}$ Showed clinical evidence supports the vision that fibroids may interfere with fertility. First, insights from the IVF model suggest a detrimental effect on implantation: the delivery rate is reduced in patients with fibroids, while it is not affected in patients who had undergone myomectomy. Second, even if randomized studies are lacking, surgical treatment appears to increase the pregnancy rate: about one of two women undergoing myomectomy for infertility subsequently conceives Understanding the crosstalk between uterine fibroids and the endometrium will provide key insights into implantation and menstrual biology and drive the development of new and innovative therapeutic options for the management of symptoms in women with uterine fibroids. ${ }^{[18]}$. The most commonly experienced adverse obstetric outcome in women with fibroids is an increase in caesarean delivery, which is mostly attributable to malpresentation. The risk of preterm labor and delivery also appears to be slightly elevated in these patients. Very large fibroids located near the placenta may place obstetric patients at slightly increased risk during labor, particularly for hysterectomy and postpartum hemorrhage. The association with placental abruption is weak and inconsistent with great variation from study to study. Neonatal outcomes do not appear different from pregnancies in women with fibroids compared with controls. ${ }^{[10],[39]}$ reported two main pieces of clinical evidence support the vision that fibroids may interfere with fertility. First, insights from the IVF model suggest a detrimental effect on implantation: the delivery rate is reduced in patients with fibroids, while it is not affected in patients who had undergone myomectomy. Second, even if randomized studies are lacking, surgical treatment appears to increase the pregnancy rate: about one of two women undergoing myomectomy for infertility subsequently conceives. Uterine fibroids, the most common neoplasm of reproductive-aged women, can have a significant impact on quality of life and may affect fertility and pregnancy outcomes. Although it is generally accepted that submucosal fibroids are of clinical significance, the effect of intramural and subserosal fibroids and the benefit of surgical removal remains an area of active debate ${ }^{[4]}$.

One more important factor considered is Alternogest. The Alternogest. Altrenogest is an orally active progestagen that inhibits gonadotropin release in a similar manner to natural progesterone. It has been successfully used to synchronize estrus cycles and to increase reproductive performance in gilts when fed at a dosage of 15-20mg for 14-18 days $^{[20],[40],[11]}$. In sows, altrenogest given at dosages of $15-30 \mathrm{mg}$ for at least 3 days starting pre- or postweaning was found to be sufficient to synchronize estrus cycles, to treat second litter depression in primiparous sows ${ }^{[20]}$ and to increase reproductive performance after early weaning ${ }^{[21]}$, Exogenous gonadotropins, such as ECG alone or in combination with hCG, have proved.

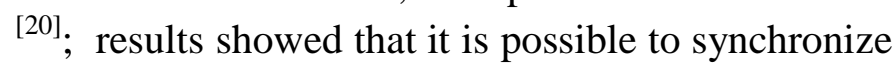
estrus and ovulation using altrenogest, ECG, and hCG or the GnRH-A D-Phe6-LHRH in sows that had not conceived at the previous mating. These treatments were able to facilitate the reintegration of the sows into a breeding schedule employing fixedtime insemination. The best results, as measured on the ovary response and reproductive performance, can be achieved with $20 \mathrm{mg}$ altrenogest per day per animal, $800 \mathrm{IU}$ hCG, and 50?g D-Phe6-LHRH. Although a 15-day-treatment with altrenogest 
realizes spontaneous luteolyis in most animals, a treatment for 18 days might be effective in more sows were proven.

The results of ${ }^{[32]}$ study showed that the most common cause of female infertility was problems in the fallopian tubes in $27.4 \%$ of the cases, while the second most common cause was infertility by « unknown causes», in the $24.5 \%$. $53.6 \%$ of respondents with infertility of unknown causes had made several attempts of Assisted Reproduction. The third most common cause was disorders of menstruation in the $20 \%$ of the cases, following infertility due to problems in the uterus in the $9.1 \%$ of the cases. Finally, in $2.7 \%$ of the participant's infertility was due to age, an additional $2.7 \%$ due to sexual disorders and the last cause was the ovarian failure, which was common for the majority of the women tested. None of the women who responded to our questionnaires was facing cervix problems. As to the habits of the participants, $45.5 \%$ were smokers and $54.5 \%$ non-smokers.

In a prospective comparative study, ${ }^{[36]}$ enrolled 103 infertile women with uterine septum as a sole cause for reproductive failure. They had had unexplained primary infertility $>2$ years and a follow-up $>12$ months. Uterine anomalies were diagnosed by means of hysterosalpingography (HSG) and 2Dtransvaginal sonography (TVS) with intrauterine saline infusion. Hysteroscopic septoplasty was performed in the early follicular phase. Pregnancy rates (PR) according to patient and septum characteristics (septum size) were the main outcome measures.

Obesity is frequently accompanied by chronic inflammation over the whole body and is always associated with symptoms that include those arising from metabolic and vascular alterations which also leads in infertility in women,

- Infectious diseases in pregnancy include TORCH complex (toxoplasmosis, syphilis, varicella-zoster, parvovirus B19, Rubella, Cytomegalovirus, Herpes infections, hepatitis infections, and human immunodeficiency virus) and other such as listeriosis, malaria and ZIKA virus are responsible several adverse maternal and fetal outcomes. Infection is related to neuropsychiatric disorders such as anxiety, schizophrenia spectrum disorders, depression, decreased weight, autoimmune thyroid diseases, self-directed violence, and violent suicide attempts in mothers. Toxoplasmosis could be an important neglected factor for endometritis, ovarian dysfunction, impaired folliculogenesis, ovarian and uterine atrophy, a decrease in reproductive organs weight and reproductive performance in women. We reviewed the role of the immunological profile such as proinflammatory cytokines and hormonal changes as main potential mechanisms related to this infection and development of maternal-fetal and reproductive disorders ${ }^{[12]}$.

\subsection{Factors Effecting Male}

Infertility and problems of impaired fecundity have been a concern through ages and is also a significant clinical problem today, which affects $8-12 \%$ of couples worldwide. Of all infertility cases, approximately $40-50 \%$ is due to "male factor" infertility and as many as $2 \%$ of all men will exhibit suboptimal sperm parameters.

Male infertility refers to a male's inability to result in pregnancy in a fertile female. "Malefactor" infertility is seen as an alteration in sperm concentration and/or motility and or morphology in at least one sample of two sperm analyzes collected 1 and 4 weeks apart. In humans, it accounts for 40 $50 \%$ of infertility and affects approximately $7 \%$ of all men. Male infertility is commonly due to deficiencies in the semen, and semen quality is used as a surrogate measure of male fecundity ${ }^{[22],[34]}$ the review summarizes the effects of more than 20 metals that, research has indicated, may influence male reproductive health. Though males lack an apparent, easily measurable reproductive cycle, progress has been made in evaluating tests to identify chemical hazards and estimate reproductive health risks. Some agents discussed in this review are well known to have potentially toxic effects on the male reproductive system, whereas some are not so well established in toxicology. This review 
attempts to cover most of the known toxicants and their effects on male fertility. The literature suggests a need for further research in those chemicals that are reactive and capable of covalent interactions in biological systems, as well as those defined as mutagens and/or carcinogens, to cause aneuploidy or other chromosomal aberrations, affect sperm motility in vitro, share hormonal activity or affect hormone action, and those that act directly or indirectly to affect the hypothalamo-pituitarygonadal axis.

The other factor studied during the past five decades, is the human sperm counts have declined and the occurrence of testicular cancer and cryptorchidism has increased. Though males lack an obvious, easily measurable reproductive cycle, and the primary clinical indicator, semen analysis, offers uncertain clues to reproductive performance, progress is being made in developing and evaluating tests to identify chemical hazards and estimate human health risks ${ }^{[30]}$. The mammalian male reproductive function can be affected through a direct effect on the testis, resulting in decreased or altered sperm production, through impairment of the accessory sex gland secretions, and indirectly through the neuroendocrine system, causing a hormonal imbalance $^{[8]}$. Adverse effects on male fertility include altered genetic material of sperm, contributing to altered spermatogenesis, pregnancy loss, or genetic disease in offspring. Common endpoints for the evaluation of male reproductive function include a size of the testis, semen quality, secretory function of the prostate and seminal vesicles, reproductive endocrine function, impotence or reduced libido, and fertility ${ }^{[28]}$. Recent evidence suggests that there may be environmental reasons for deteriorating sperm quality, including occupational exposure to various metals, chemicals, heat, and radiation. In addition, exposure to pesticides has been linked to alterations in spermatogenesis $^{[37]}$.

When assessing reproductive effects of a certain metal on male reproductive health, one must make an allowance for possible influences of concomitant exposures to other toxic and essential metals; these may act additively, synergistically, or antagonistically. Certain toxic metals, such as lead and cadmium, are pervasive in the human environment and accumulate in the human body over a lifetime; biomarkers of lead and cadmium exposure commonly correlate with age, smoking habits, and alcohol consumption ${ }^{[24]}$.

The result from different scientific studies indicated that the degree of toxic manifestation of different metals depends on dose, duration, route of administration, and other physiological factors, especially nutrition. Toxic manifestation by different metals varies from species to species. The signs and symptoms of metal toxicity depend on the duration of exposure, type of metal, the condition of the workplace, socioeconomic status, and history of the disease. The extensive literature study has explored that there is a gap of knowledge in the proper toxicity survey. Existing ongoing trials will provide answers on the safety and effectiveness of exposure of these metals, and further efforts should be made to widen our knowledge in this unmapped area of research ${ }^{[34]}$.

A study Published by Oxford University Press on behalf of the European Society of Human Reproduction and Embryology observed 7,2014 populations from different countries and defined different reproductive states: 'infertility' (a delay in conception for a given period of time), 'subfecundity' (a delay in conception or difficulty in carrying a pregnancy to term) and 'childlessness' (no child after a given period of marriage) ${ }^{[40]}$ Updated projections of infertility in the United States: 1995 From less developed countries the word 'childlessness' definition was often used in reports, A report of 28 countries in sub-Saharan Africa, estimated a single weighted average percentage infertility with weight proportional to the number of women aged 20-44 in marital and consensual unions in each country. The introduction of intracytoplasmic sperm injection (ICSI) has made it possible for men with severe oligozoospermia and azoospermia to father children, but the genetic risks of this highly invasive technique must be considered. These include the risks of transferring the cystic 
fibrosis conductance regulator (CFTR) gene, somatic and sex chromosome abnormalities, and microdeletions of the Y chromosome. ${ }^{[3]}$

Studies that examine differences in semen quality between geographically diverse cohorts may help identify such factors. An ongoing network of international studies, begun in 1997, was designed to address this question. In these collaborative prospective studies, the use of common study protocols, analytic methods, and quality control procedures should minimize extraneous inter study differences. They should provide unbiased estimates of variability among cities that have been reported to differ widely in semen quality, provide baseline levels of male biomarkers for future studies, and generate hypotheses of environmental causes of variation in these parameters $^{[42]}$.

[31], over a period, noted that declining semen quality in the entire population as well as in selected men. Indeed, a significant decrease in both sperm concentration and total sperm count, and a severe decline of rapid progressive motility (grade a) were observed in the two populations. ${ }^{[42]}$, postulate that the percentage of spermatozoa with grade ' $a$ ' motility is the strongest indicator of the potential for in vivo fertilizing capacity of semen. Their results suggested that an increasing proportion of men in their region could be candidates for assisted medical procreation techniques over time.

Sperm morphology seems to be a very sensitive parameter of exposure to the toxicants. Any of them should lead to a temporary or permanent decrease in normal sperm morphology. As expected, semen parameters in the population with normal sperm count declined less severely than in the total population. ${ }^{[31]}$.A man's age and the duration of his sexual abstinence before the collection of semen influence the characteristics of the semen. Older age contributed significantly to the decreases in sperm concentration, the percentage of motile sperm, and the percentage of normal spermatozoa. ${ }^{[38]}$

Screening of males by semen analysis provides some insight about the underlying pathological problems occurring in the male genital tract. ${ }^{[23]}$ found that of total 3084 cases 1104 (35.80\%) males had normal sperm count and rest 1980 (64.20\%) males had abnormal semen analysis report. This is similar to a study done in 2012 which reported the incidence of male infertility as $62 \% 24$. The reported prevalence of oligozoospermic, azoospermic and asthenoteratozoospermic in cases of primary infertility in the same study was $33.17 \%, 9.89 \%$, and $1.08 \%$ respectively.

Another important factor is obesity being overweight or obese and sperm parameters, as well as proinflammatory cytokine levels in semen plasma $^{[13]}$, collected semen samples from 272 donors, including 82 normal weight, 150 overweight, and 40 obese individuals respectively. Based on the sperm parameters measured by CASA, they found a negative relationship between sperm concentration, motility, and BMI, which indicated that both overweight and obese males were associated with low sperm counts and declines in sperm motility. Moreover, the concentrations of IL- 6 and TNF- $\alpha$ were measured in the semen plasma and the results clearly showed that their levels significantly increased in the semen plasma from obese or overweight males compared with that of normal body weight individuals. These observations further demonstrated that obesity or overweight can indeed up-regulate cytokine concentrations in the male genital tract and impair sperm quality.

[5], studied ob Reactive oxygen species (ROS) OS, defined as a physiological redox imbalance, occurs due to either an overabundance of ROS or a deficiency of antioxidants. Infertile men can have in their seminal plasma, elevated levels of ROS and decreased antioxidant concentrations as a cause of male infertility. Excessive levels of ROS coupled with a deficiency in antioxidants can lead to oxidative stress (OS), which in turn can lead to nuclear and mitochondrial DNA damage, telomere shortening, epigenetic alterations, and $\mathrm{Y}$ chromosomal microdeletions. In this review, we discuss how OS induces DNA damage and the types of DNA damage that can occur. We also briefly touch on the clinical consequences of OS-induced DNA damage. 
Another factor to be considered is the Herpes simplex virus (HSV) is commonly present in the semen of male seeking fertility evaluation. ${ }^{[43]}$, demonstrated some important associations between the presence of HSV in semen and changes in seminal parameters. Therefore, screening for HSV in semen should be considered for diagnostic settings in unexplained infertility, in assisted reproduction, and for donor semen to prevent unnecessary viral transmission to the female genital tract. Further studies enrolling a larger number of patients are necessary to confirm these data and to elucidate the clinical relevance of HSV presence in semen.

\subsection{Consequences of Infertility}

1. Second marriage is the first thing which comes into mind in remote areas and in families which give importance to the upcoming generations as they heir.

2. Adoption is another consequence. In the sample, 10 percent of the women had adopted a child or children, and another 12 percent stated their intention of adopting at a future date. The process of adoption had been very informal, wiling legal formalities. Generally, the adopted child was the child of a close relative, such as the brother-in-law or the sister of the woman. Adoption was considered to have taken place simply by bringing the child to the house. If for any reason the child was unable to adjust, or there were other problems, the adoption could be terminated merely by sending the child back to his or her birth parents. Most of the women who had postponed adoption to a later date said they wanted to wait and see whether they could conceive and they were quite hopeful of this (39 percent of them). They were ready to adopt in the future if nothing happened. Around 13 percent had not adopted because there was not a child available for them to adopt. Some women (10 percent) said their husbands disapproved ${ }^{[28]}$.

3. The relationship between couples becomes hard work, and unwanted challenges will be encountered along the way. They cannot help themselves manage difficulties that arise in their union and take a hasty decision of quitting the world.

\section{Conclusions}

Infertility is a global health issue affecting couples, not all couples who desire a pregnancy will achieve one spontaneously and a proportion of couples will need medical help to resolve underlying fertility problems and also both of them are to be taken into account instead of focusing on only women. There are many ways available in modern medical science to try IVF, IUI.ICSI, IMSI, etc though they take time and money they make us lucky after a long wait, apart from all this, an important thing needed is emotional support and a positive attitude to think that they can improve their fertility and achieve success in their lives to have a baby.

\section{Acknowledgement}

I wish to thank my professors and colleagues who have provided me with literature.

\section{References}

1. Arendell, T. (2000). Conceiving and investigating motherhood: The decade's scholarship. Journal of Marriage and Family. $\quad$ https://doi.org/10.1111/j.17413737.2000.01192.x

2. Black, K. I., Gupta, S., Rassi, A., \& Kubba, A. (2010). Why do women experience untimed pregnancies? A review of contraceptive failure rates. Best Practice and Research: Clinical Obstetrics and Gynaecology. https://doi.org/10.1016/j.bpobgyn.2010.02.0 02

3. Boivin, J., Bunting, L., Collins, J. A., \& Nygren, K. G. (2007). International estimates of infertility prevalence and treatment-seeking: potential need and demand for infertility medical care. 22(6), 1506-1512.

4. Brady, P. C., Stanic, A. K., \& Styer, A. K. (2013). Uterine fibroids and subfertility: an update on the role of myomectomy. 255-259. 
https://doi.org/10.1097/GCO.0b013e328361 2188

5. Bui, A. D., Sharma, R., \& Henkel, R. (2018). Reactive oxygen species impact on sperm DNA and its role in male infertility. (February), $1-10$. https://doi.org/10.1111/and.13012

6. Carmeli, Y. S., \& Birenbaum-Carmeli, D. (1994). The predicament of masculinity: Towards understanding the male's experience of infertility treatments. Sex Roles. https://doi.org/10.1007/BF01544669

7. Casini, M. L., Rossi, F., Agostini, R., \& Unfer, V. (2006). Effects of the position of fibroids on fertility. 22(February), 106-109. https://doi.org/10.1080/09513590600604673

8. Chandra, A. K., Goswami, H., \& Sengupta, P. (2012). Dietary calcium-induced cytological and biochemical changes in the thyroid. Environmental Toxicology and Pharmacology, 34(2), 454-465.

9. Chodorow, N. J. (1989). Feminism and psychoanalytic theory. Yale University Press.

10. Endocrinology, R. (2008). Reviews Fibroids and reproductive outcomes: a systematic literature review from conception to delivery. (April), 357-366. https://doi.org/10.1016/j.ajog.2007.12.039

11. Estienne, M. J., Harper, A. F., Horsley, B. R., Estienne, C. E., \& Knight, J. W. (2001). Effects of PG 600 on the onset of estrus and ovulation rate in gilts treated with Regumate. Journal of Animal Science, 79(11), 2757-2761.

12. Fallahi, S., Rostami, A., Shiadeh, M. N., Behniafar, H., \& Paktinat, S. (2017). ScienceDirect An updated literature review on maternal-fetal and reproductive disorders of Toxoplasma gondii infection. Journal of Gynecology Obstetrics and Human Reproduction.

https://doi.org/10.1016/j.jogoh.2017.12.003

13. Fan, W., Xu, Y., Liu, Y., Zhang, Z., Lu, L., \& Ding, Z. (2018). Obesity or Overweight, a
Chronic Inflammatory Status in Male Reproductive System, Leads to Mice and Human Subfertility. 8(January), 1-11. https://doi.org/10.3389/fphys.2017.01117

14. Ferreira, M., Vicente, S., Duarte, J., \& Chaves, C. (2015). Quality of Life of Women with Infertility. Procedia - Social and Behavioral Sciences, 165, 21-29. https://doi.org/10.1016/j.sbspro.2014.12.600

15. Fisch, B., Kraicer, R. K., Amit, S., Zukerman, Z., Ovadia, J., \& Tadir, Y. (1990). The relationship between sperm parameters and fertilizing capacity in vitro: a predictive role for swim-up migration. Journal of In Vitro Fertilization and Embryo Transfer, 7(1), 38-44.

16. Garey, A. I., \& Arendell, T. (2001). Children, Work, and Family. Working Families: The Transformation of the American Home, 293.

17. Glenn, E. N., Chang, G., \& Forcey, L. R. (2016). Mothering: Ideology, experience, and agency. Routledge.

18. Ikhena, D. E., \& Bulun, S. E. (2017). Literature Review on the Role of Uterine Fibroids in Endometrial Function. https://doi.org/10.1177/1933719117725827

19. Josselson, R. (1998). Revising herself: The story of women's identity from college to midlife. Oxford University Press.

20. Kauffold, J., Beckjunker, J., Kanora, A., \& Zaremba, W. (2007). Synchronization of estrus and ovulation in sows not conceiving in a scheduled fixed-time insemination program. Animal Reproduction Science. https://doi.org/10.1016/j.anireprosci.2006.01 .004

21. Koutsotheodoros, F., Hughes, P. E., Parr, R. A., Dunshea, F. R., Fry, R. C., \& Tilton, J. E. (1998). The effects of post-weaning progestagen treatment (Regumate) of earlyweaned primiparous sows on subsequent reproductive performance. Animal Reproduction Science, 52(1), 71-79.

22. Kumar, N., Choudhari, A. R., \& Singh, A. 
K. (2015). Prevalence of Male Factor Infertility in Last Ten Years at a Rural Tertiary Care Centre of Central India: A Retrospective Analysis. 2(3), 132-136. https://doi.org/10.5958/2394-

2754.2015.00002.8

23. Kumar, N., \& Singh, A. (2016). Trends of male factor infertility, an important cause of infertility: A review of the literature. Journal of Human Reproductive Sciences, 8(4), 191. https://doi.org/10.4103/0974-1208.170370

24. Lin, Y.-S., Caffrey, J. L., Chang, M.-H., Dowling, N., \& Lin, J.-W. (2010). Cigarette smoking, cadmium exposure, and zinc intake on obstructive lung disorder. Respiratory Research, 11(1), 53.

25. Macro, O., Organization, W. H., DHS, S., \& reports, C. (2004). Infecundity, infertility, and childlessness in developing countries. Surveys (DHS) Comparative Reports. Retrieved from https://scholar.google.com/scholar?hl=en\&a s_sdt $=0 \% 2 \mathrm{C} 5 \& \mathrm{q}=$ World + Health+Organizati on.+Infecundity\%2C+Infertility\%2C+and+ Childlessness + in + Developing + Countries. $+\mathrm{D}$

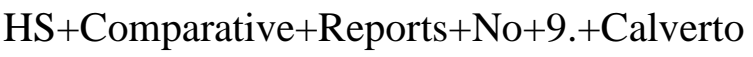
$\mathrm{n} \% 2 \mathrm{C}+$ Maryland\%2C+USA\%3A+ORC+M acro+and+the+World+Health+Organi

26. McMahon, M. (1995). Engendering motherhood: Identity and self-transformation in women's lives. Guilford Press.

27. Morton, H., Rolfe, B., Clunie, G. A., Anderson, M., \& Morrison, J. (1977). AN EARLY PREGNANCY FACTOR DETECTED IN HUMAN SERUM BY THE ROSETTE INHIBITION TEST. The Lancet, 309(8008),

394-397.

https://doi.org/10.1016/S0140-

6736(77)92605-8

28. Nordberg, G. F., Fowler, B. A., \& Nordberg, M. (2014). Handbook on the Toxicology of Metals. Academic Press.

29. Pradesh, A. (1998). The study.

30. Queiroz, E. K. R. de, \& Waissmann, W. (2006). Occupational exposure and effects on the male reproductive system. Cadernos de Saúde Pública, 22(3), 485-493.

31. Romain, F., Achard, V., Geoffroy-siraudin, C., \& Dieudonne, A. (2012). Decline of semen quality among 10932 males consulting for couple infertility over a 20 year period in Marseille, France. (September 2011), 584-590. https://doi.org/10.1038/aja.2011.173

32. Roupa, Z., Polikandrioti, M., Sotiropoulou, P., Faros, E., Koulouri, A., Wozniak, G., \& Gourni, M. (2009). CAUSES OF INFERTILITY IN WOMEN AT REPRODUCTIVE AGE. 2, 80-87.

33. Ruddick, S. (1994). Thinking mothers/conceiving birth. Yale University Press.

34. Sengupta, P. (2013). nvironmental and occupational exposure of metals and their role in male reproductive functions. 36(June 2012),

353-368. https://doi.org/10.3109/01480545.2012.7106 31

35. Sereno, S., Leal, I., \& Maroco, J. (2009). Construção e validação de um questionário de valores e crenças sobre sexualidade, maternidade e aborto. Psicologia, Saúde \& Doenças, 10(2), 193-204.

36. Shokeir, T., Abdelshaheed, M., El-shafie, M., Sherif, L., \& Badawy, A. (2011). European Journal of Obstetrics \& Gynecology and Reproductive Biology Determinants of fertility and reproductive success after hysteroscopic septoplasty for women with unexplained primary infertility: a prospective analysis of 88 cases. European Journal of Obstetrics and Gynecology, 155(1), 54-57. https://doi.org/10.1016/j.ejogrb.2010.11.015

37. Sinclair, S. (2000). Male infertility: nutritional and environmental considerations. Alternative Medicine Review: A Journal of Clinical Therapeutic, 5(1), 28-38.

38. Society, M. M. (1995). , m.d., p. 332(5). 
39. Somigliana, E., Vercellini, P., Daguati, R., Pasin, R., Giorgi, O. De, \& Crosignani, P. G. (2007). Fibroids and female reproduction : a critical analysis of the evidence. 13(5), 465476.

40. Stephen, E. H., \& Chandra, A. (1998). Updated projections of infertility in the United States: 1995-2025. Fertility and Sterility, 70(1), 30-34.

41. Stevenson, J. S., \& Davis, D. L. (1982). Estrous synchronization and fertility in gilts after 14-or 18-day feeding of altrenogest beginning at estrus or diestrus. Journal of Animal Science, 55(1), 119-123.

42. Swan, S. H., Elkin, E. P., \& Fenster, L. (2000). The Question of Declining Sperm Density Revisited: An Analysis of 101 Studies Published 1934 - 1996. 108(10), 961-966.

43. Takeda, K. I., Piva, S., Piva, J. P., Irie, M. M. T., \& Consolaro, M. E. L. (2017). Affiliations: Professor Marcia Edilaine Lopes Consolaro, Ph.D. Urology. https://doi.org/10.1016/j.urology.2017.11.05 0

44. Van Balen, F. (1996). Child-rearing following in vitro fertilization. Journal of Child Psychology and Psychiatry, 37(6), 687-693. 